\title{
A SPECTROSCOPICALLY CONFIRMED DOUBLE SOURCE PLANE LENS SYSTEM IN THE HYPER SUPRIME-CAM SUBARU STRATEGIC PROGRAM
}

\author{
Masayuki Tanaka ${ }^{1}$, Kenneth C. Wong $^{1}$, Anupreeta More ${ }^{2}$, Arsha Dezuka ${ }^{3}$, Eirchi Egami ${ }^{4}$, Masamune Oguri ${ }^{2,5,6}$, \\ Sherry H. Suyu ${ }^{7,8}$, Alessandro Sonnenfeld ${ }^{2}$, Ryo Higuchi ${ }^{9}$, Yutaka Komiyama ${ }^{1}$, Satoshi Miyazaki ${ }^{1,10}$, \\ Masafusa Onoue ${ }^{1,10}$, Shuri Oyamada ${ }^{11}$, and Yousuke Utsumi ${ }^{12}$ \\ ${ }^{1}$ National Astronomical Observatory of Japan, Osawa 2-21-1, Mitaka, Tokyo 181-8588, Japan \\ ${ }^{2}$ Kavli Institute for the Physics and Mathematics of the universe (Kavli IPMU, WPI), University of Tokyo, Chiba 277-8583, Japan \\ ${ }^{3}$ Department of Astronomy, University of Kyoto, Kitashirakawa-Oiwake-cho, Sakyo-ku, Kyoto 606-8502, Japan \\ ${ }^{4}$ Steward Observatory, University of Arizona, 933 North Cherry Avenue, Tucson, AZ 85721, USA \\ ${ }^{5}$ Department of Physics, University of Tokyo, 7-3-1 Hongo, Bunkyo-ku, Tokyo 113-0033, Japan \\ ${ }^{6}$ Research Center for the Early universe, University of Tokyo, Tokyo 113-0033, Japan \\ ${ }^{7}$ Max Planck Institute for Astrophysics, Karl-Schwarzschild-Strasse 1, D-85740 Garching, Germany \\ ${ }^{8}$ Institute of Astronomy and Astrophysics, Academia Sinica, P.O. Box 23-141, Taipei 10617, Taiwan \\ ${ }^{9}$ Institute for Cosmic Ray Research, The University of Tokyo, 5-1-5 Kashiwa-no-Ha, Kashiwa City, Chiba, 277-8582, Japan \\ ${ }^{10}$ SOKENDAI (The Graduate University for Advanced Studies), Mitaka, Tokyo 181-8588, Japan \\ ${ }^{11}$ Japan Women's University, 2-8-1 Mejirodai, Bunkyo, Tokyo 112-8681, Japan \\ ${ }^{12}$ Hiroshima Astrophysical Science Center, Hiroshima University, 1-3-1, Kagamiyama, Higashi-Hiroshima, Hiroshima 739-8526, Japan \\ Received 2016 June 11; revised 2016 June 29; accepted 2016 June 29; published 2016 July 25
}

\begin{abstract}
We report the serendipitous discovery of HSC J142449-005322, a double source plane lens system in the Hyper Suprime-Cam Subaru Strategic Program. We dub the system Eye of Horus. The lens galaxy is a very massive early-type galaxy with stellar mass of $\sim 7 \times 10^{11} M_{\odot}$ located at $z_{\mathrm{L}}=0.795$. The system exhibits two arcs $/$ rings with clearly different colors, including several knots. We have performed spectroscopic follow-up observations of the system with FIRE on Magellan. The outer ring is confirmed at $z_{\mathrm{S} 2}=1.988$ with multiple emission lines, while the inner arc and counterimage is confirmed at $z_{\mathrm{S} 1}=1.302$. This makes it the first double source plane system with spectroscopic redshifts of both sources. Interestingly, redshifts of two of the knots embedded in the outer ring are found to be offset by $\Delta z=0.002$ from the other knots, suggesting that the outer ring consists of at least two distinct components in the source plane. We perform lens modeling with two independent codes and successfully reproduce the main features of the system. However, two of the lensed sources separated by $\sim 0.7$ arcsec cannot be reproduced by a smooth potential, and the addition of substructure to the lens potential is required to reproduce them. Higher-resolution imaging of the system will help decipher the origin of this lensing feature and potentially detect the substructure.
\end{abstract}

Key words: galaxies: individual (HSC J142449-005322) - gravitational lensing: strong

\section{INTRODUCTION}

Strong gravitational lensing is a powerful tool to map the matter distribution around galaxies on small scales. In particular, it is possible to infer the total mass enclosed within the Einstein radius, allowing us to separate the dark and baryonic mass distribution of massive galaxies and constrain the stellar initial mass function (IMF; e.g., Treu et al. 2010). However, these measurements are complicated by various model degeneracies.

Lenses with multiple sources at distinct redshifts offer a unique opportunity to break these degeneracies, leading to accurate measurements of the dark matter distribution and IMF (e.g., Sonnenfeld et al. 2012). In addition, such double source plane (DSP) lens systems can be a useful, complementary cosmological probe (e.g., Collett et al. 2012; Collett \& Auger 2014; Linder 2016), provided the mass-sheet degeneracy is broken (e.g., Falco et al. 1985; Schneider 2014). However, DSP lenses are extremely rare. Only a handful are currently known (e.g., Gavazzi et al. 2008; Tu et al. 2009; Cooray et al. 2011; Brammer et al. 2012), and spectroscopic redshifts of the second sources have not been measured for these systems due to their faintness. Ongoing/upcoming wide-area imaging surveys are expected to increase the number of such rare but fruitful systems. Here, we report the discovery of a DSP lens system from the Hyper Suprime-Cam Subaru Strategic Program (hereafter the HSC survey).

The HSC survey is the largest observing program ever approved at the Subaru $8.2 \mathrm{~m}$ telescope (300 nights). With a three-layered survey strategy over $\sim 1400 \mathrm{deg}^{2}$, it aims to address several outstanding astrophysical questions such as the nature of dark energy, galaxy evolution, and cosmic reionization. The survey is $\sim 20 \%$ complete as of this writing. While the survey is still in an early phase, the exquisite data quality has led to the exciting discovery of this DSP lens. The system, HSC J142449-005322, was serendipitously discovered at a science school for undergraduate students hosted at the National Astronomical Observatory of Japan in 2015. While visually inspecting galaxy cluster candidates, we found a likely cluster member with a clear sign of gravitational lensing. A more careful inspection revealed the multi-source nature of the system. We dub it Eye of Horus, as the system resembles it.

We describe our photometric and spectroscopic data in Section 2. We discuss properties of the lens galaxy in Section 3 and describe our lens modeling procedures in Section 4. Our main results are presented in Section 5. We adopt $\mathrm{H}_{0}=70 \mathrm{~km} \mathrm{~s}^{-1} \mathrm{Mpc}^{-1}, \Omega_{M}=0.27$, and $\Omega_{\Lambda}=0.73$. Magnitudes are $\mathrm{AB}$ magnitudes. 


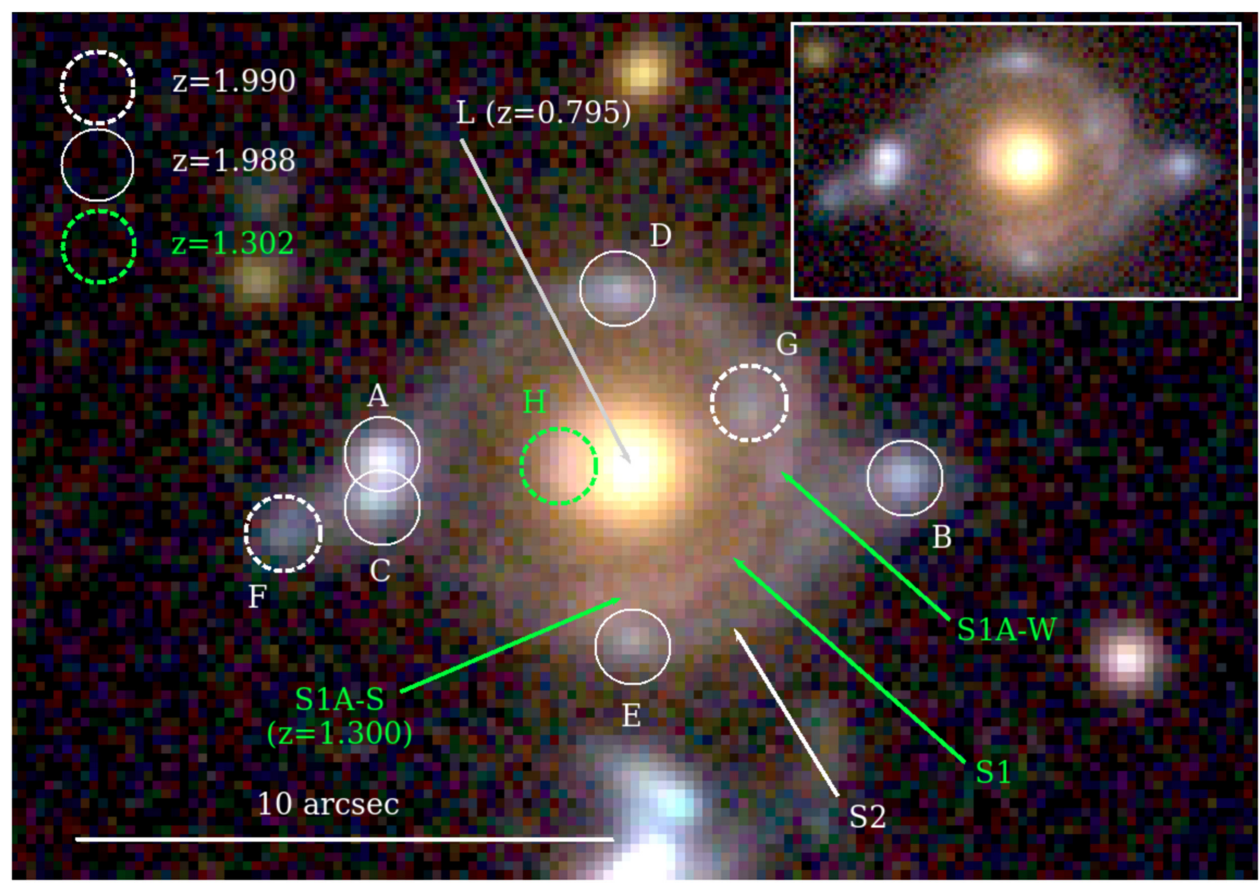

Figure 1. riz color composite of Eye of Horus. The lens galaxy is labeled "L," and the inner and outer sources are labeled "S1" and "S2," respectively. The knots in the sources are labeled alphabetically in approximate order of brightness. Note that the two rings clearly have different colors. The spectroscopic redshifts of the various features are indicated by the colored circles in the upper left. The angular scale is shown by the bar at the bottom left. The inset shows the lens system with no labels overlaid.

\section{DATA}

In this section, we describe the photometric and spectroscopic properties of the system. The lens is located at (R.A., decl. $)=\left(14^{\mathrm{h}} 24^{\mathrm{m}} 49^{\mathrm{s}} .0,-00^{\circ} 53^{\prime} 21^{\prime \prime} 65\right)(\mathrm{J} 2000)$. A multicolor image of Eye of Horus constructed from HSC imaging data is shown in Figure 1. The two sources with distinct colors are clearly seen around the lens: a red inner source (S1) and a blue Einstein ring (S2). These arcs are approximately $3^{\prime \prime}$ in radius. Several knots with similar colors are embedded in the arcs, which are useful features to constrain our lens models (Section 4).

\subsection{Photometric Data}

Eye of Horus is located in the Wide layer of the HSC survey, which aims to cover $\sim 1400 \mathrm{deg}^{2}$ down to $r \sim 26$. This Letter is based on the S15A internal data release. The data are processed with hscPipe v3.8.5, a version of Large Synoptic Survey Telescope pipeline (Ivezic et al. 2008; Axelrod et al. 2010) calibrated against Pan-STARRS1 (Schlafly et al. 2012; Tonry et al. 2012; Magnier et al. 2013). A summary of the HSC data on this system is given in Table 1. The exposure times are short, but reach fairly deep thanks to the superb image quality. We find that the system has also been observed by KiDS (de Jong et al. 2013) and VISTA-VIKING (Edge et al. 2013). However, these shallower data do not reveal the double source nature of the system-only the outer arc is seen in the KiDS data, and VISTA-VIKING detect only the few brightest knots. We use only the photometric data from HSC in this Letter.

\subsection{Spectroscopic Data}

The lens galaxy has a spectroscopic redshift of $z_{\mathrm{L}}=0.795$ from the Sloan Digital Sky Survey (Alam et al. 2015), and we discuss its properties in Section 3. In order to determine the
Table 1

Photometric Data

\begin{tabular}{lccc}
\hline \hline Filter & Seeing $\left({ }^{\prime \prime}\right)$ & ${\text { Exposure Time }(\mathrm{s})^{\mathrm{a}}}^{\mathrm{a}}$ & $5 \sigma$ Depth $^{\mathrm{b}}$ \\
\hline$g$ & 0.81 & 450 & 26.7 \\
$r$ & 0.62 & 450 & 26.4 \\
$i$ & 0.61 & 1600 & 26.4 \\
$z$ & 0.66 & 1600 & 25.5 \\
$y$ & 0.66 & 2000 & 24.6 \\
\hline
\end{tabular}

Notes.

${ }^{\text {a }}$ Some of the $y$-band data are taken under poor conditions.

b $5 \sigma$ depth for point sources. The depths within 1 ." 5 apertures, which may be more relevant for extended sources, are shallower by $\sim 0.2 \mathrm{mag}$.

redshifts of the background sources, we performed follow-up spectroscopic observations with the Folded-port Infrared Echellette (FIRE; Simcoe et al. 2013) on the $6.5 \mathrm{~m}$ Magellan I Baade telescope. FIRE covers the wavelength range $0.82-2.51 \mu \mathrm{m}$ at a spectral resolution of $R=6000$. The observations were taken on the nights of UT 2016 February 15-16. Conditions were clear, and the seeing varied from 0 ". 6 to 1 !" 0 . A slit width of $1^{\prime \prime}$ was used to image the primary features of S1 and S2 across six separate pointings with exposure times ranging from 10 to 40 minutes. The data are reduced using a combination of the FIREHOSE pipeline and custom IDL scripts.

We successfully determine the redshifts of most of the main features of both sources indicated in Figure 1, making this the first DSP lens with spectroscopic confirmation of both source redshifts. As shown in Figure 2, we measure the redshift of the outer source to be $z_{\mathrm{S} 2}=1.988$ based on multiple detections of the $\left[\mathrm{O}_{\mathrm{II}}\right], \mathrm{H} \beta,\left[\mathrm{O}_{\mathrm{III}}\right]$, and $\mathrm{H} \alpha$ lines. Interestingly, $\mathrm{S} 2$ has two components that are slightly offset by $\Delta z \approx 0.002$ in redshift 

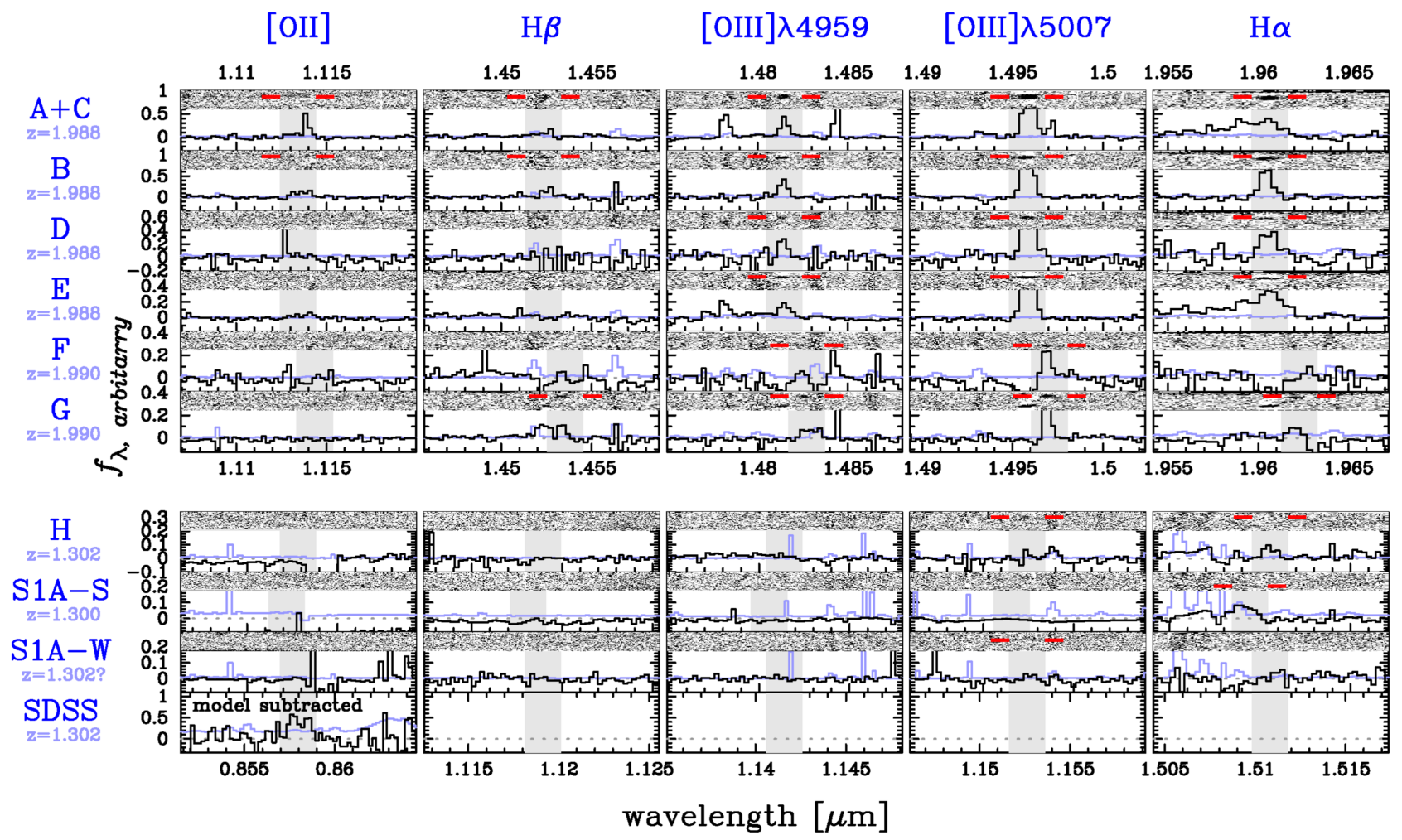

Figure 2. 1D and 2D spectra of the arcs and knots taken with FIRE. The black and light blue spectra are the 1D object and noise spectra, respectively, and are binned over $2 \AA$. The shaded regions show where strong emission lines are located. Not all the lines are securely detected and we mark in red in the $2 \mathrm{D}$ spectra the secure or likely line detections. Note the small redshift offset of $\mathrm{F}$ and $\mathrm{G}$ with respect to A-E. There is also a small offset between $\mathrm{H}$ and S1A-S. The bottom panel shows the spectrum of the lens from SDSS with the best-fit continuum spectrum subtracted. The [O II] emission from $\mathrm{H}$ is observed (see the text for details).

space. However, they are likely physically associated given the small difference.

The inner source is confirmed at $z_{\mathrm{S} 1}=1.302$ with weaker detections of the $[\mathrm{O}$ III] and $\mathrm{H} \alpha$ lines. In addition to our FIRE spectra, the spectrum from SDSS also confirms the redshift of $\mathrm{S} 1$-feature $\mathrm{H}$ is included within the SDSS fiber targeting the lens galaxy and we observe clear [O II] emission from $\mathrm{H}$ once the continuum is subtracted. S1 also seems to have two components as indicated by the small redshift offset of $\Delta z \approx 0.002$ between $\mathrm{H}$ and the southern segment of the arc (S1A-S) as well as a very marginal hint of the western segment (S1A-W) being at a redshift consistent with $\mathrm{H}$, suggesting that S1A-W is the counterimage of $\mathrm{H}$. Further follow-up observations will reveal the detailed dynamics of each source.

\section{LENS GALAXY PROPERTIES}

The lens galaxy is a passive galaxy with no clear signs of active star formation based on the SDSS spectrum. Preliminary results of the CAMIRA algorithm (Oguri 2014) applied to the HSC survey data suggest that there is a rich cluster of galaxies at $z_{\text {phot }} \sim 0.81$ at the location of the lens. The richness of $N \sim 50$ implies a cluster mass of $M_{\mathrm{vir}} \sim 7 \times 10^{14} M_{\odot}$. Given the lens galaxy's large stellar mass (estimated below) and proximity to the cluster center, it is potentially the brightest cluster galaxy. A more detailed analysis of the lens environment based on multi-object spectroscopy from the Inamori Magellan Aerial Camera and Spectrograph (IMACS; Dressler et al. 2011) will be presented in a follow-up work (K. C. Wong et al., in preparation).
Despite the superb seeing, the lens and the surrounding arcs are still blended in the HSC imaging. In order to study the lens and perform detailed analyses of the background sources, we fit the lens with a double Sérsic model and subtract it from the images using GleE (Suyu \& Halkola 2010; Suyu et al. 2012). We first fit the lens in the $i$-band, in which the lens is detected at a high signal-to-noise ratio of $\sim 500$ within a 1 ." 5 aperture and the seeing is good (0."61; see Table 1). The PSF is modeled in each exposure and is evaluated at the position of the lens by coadding the PSF models. We then fit the lens in the other bands with the structural parameters such as centroid, effective radius, ellipticity, and position angle fixed to those measured in the $i$-band. Only the flux normalization is allowed to vary. The PSF model in each band is evaluated in the same way as the $i$ band. The multicolor lens-subtracted image is shown in the left panel of Figure 3. An arc-like feature $(\mathrm{H})$ on the left of the lens emerges. The images show no subtraction residuals at the location of the lens, demonstrating our accurate modeling of PSF and the lens galaxy light.

Using the five-band photometry and the known redshift of the lens galaxy, we fit its spectral energy distribution (SED) using MIZUKI (Tanaka 2015). Due to the strong blending, we use the double Sersic fit to the lens to estimate the flux in each band. The fit has a very extended envelope out to large radii, but we truncate the model at a radius of 3." $3(\sim 25 \mathrm{kpc}$ at the lens redshift), which is the radius of the S2 arc. This is a somewhat arbitrary choice, but it should include most of the lens light and also represent the enclosed stellar mass while keeping the flux from the (uncertain) envelope relatively small. The SED is then fit with a suite of model templates generated by Bruzual \& Charlot (2003) models assuming a Chabrier 

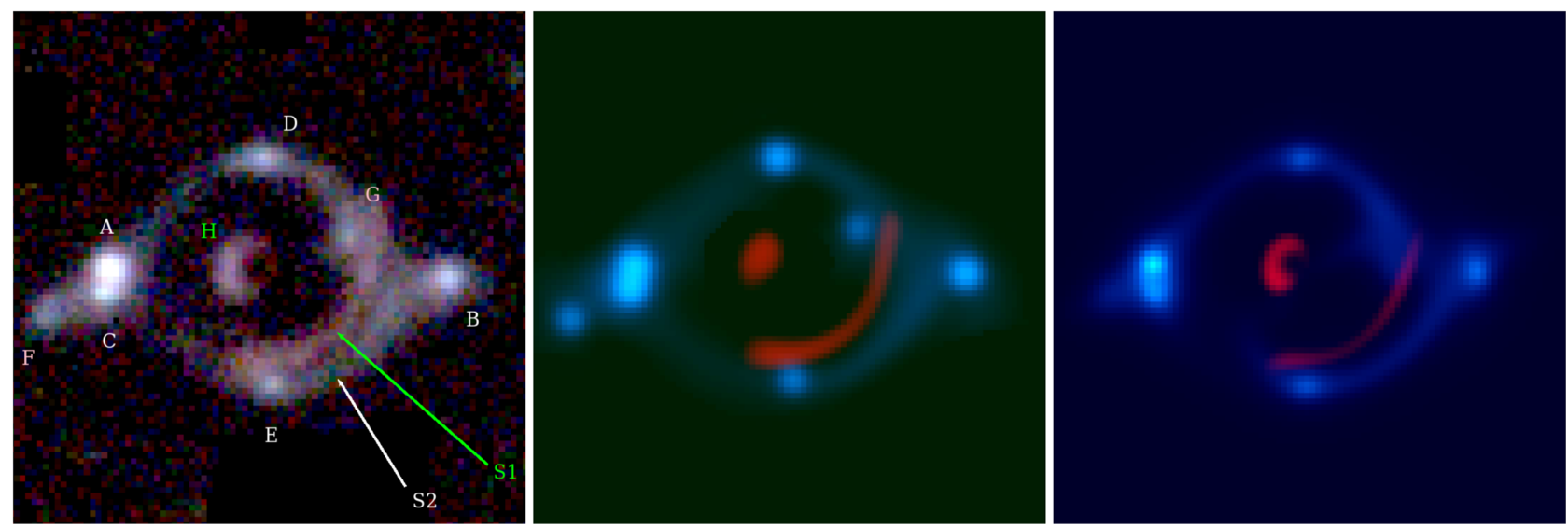

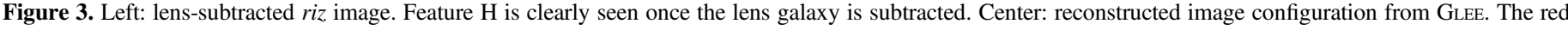

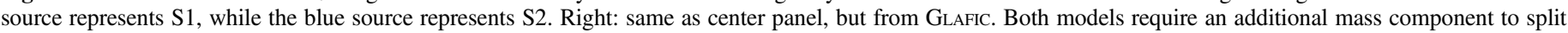
features $\mathrm{A}$ and $\mathrm{C}$ into two distinct components.

(2003) IMF and Calzetti et al. (2000) dust attenuation curve. We apply Bayesian priors on the physical properties of galaxies to keep the model parameters within realistic ranges and to effectively let the templates evolve with redshift in an observationally motivated way (for more details, see Tanaka 2015). We find that the lens galaxy is very massive -it has a stellar mass of $6.6_{-0.1}^{+0.7} \times 10^{11} M_{\odot}$, making it among the most massive galaxies around this redshift (Muzzin et al. 2013). The star formation rate from the fit is very small for its stellar mass $\left(\mathrm{SFR}=0.9_{-0.1}^{+1.3} M_{\odot} \mathrm{yr}^{-1}\right.$ ), as is the dust attenuation $\left(\tau_{V}=0.2_{-0.1}^{+0.2}\right)$. Together with the early-type morphology, the lens may be the brightest cluster galaxy. Note that the given uncertainties are just statistical uncertainties and do not account for systematic effects.

\section{LENS MODELS}

We model the system based on the data in hand using two independent codes: GleE (Suyu \& Halkola 2010; Suyu et al. 2012) and Glafic (Oguri 2010). The system is complex, and our goal is to reproduce the main features of the image configuration of both sources to obtain a basic understanding of the system. More detailed lens models will be presented in a future paper (K. C. Wong et al., in preparation).

\subsection{Pixelated Source Modeling}

We first model Eye of Horus with GLEE using pixelated grids with curvature regularization for the extended sources (Suyu et al. 2006). Lensing mass distributions are described by parameterized profiles. Model parameters of the lens and source are constrained through Markov Chain Monte Carlo sampling. We use the full multiplane lens equation (e.g., Blandford \& Narayan 1986) to calculate deflection angles since there are multiple lens and source planes involved (S. Suyu et al., in preparation).

Our modeling strategy is to first use only S1 to constrain the parameters of the main lens galaxy (L), then to model L and S1 simultaneously with the light from $\mathrm{S} 2$ as constraints. We separate $\mathrm{S} 1$ and $\mathrm{S} 2$ by subtracting the HSC images in two filters (after matching the seeing) weighted such that the light from either S1 or S2 is removed and leaving only light from the other. We find that using the $i$ and $z$ bands give the cleanest separation, as these filters straddle the $4000 \AA$ break of S1 and probe the UV continuum of S2. The lens galaxy L is parameterized as a singular power-law elliptical mass distribution (Barkana 1998). We fix the centroid to the galaxy light centroid and adopt flat priors on the ellipticity, orientation, and mass profile slope. We do not include external shear, as it does not drastically improve the fit.

For the second part of our modeling, S1 is parameterized as a singular isothermal ellipsoid (SIE) with flat priors on its ellipticity and orientation. The centroid of S1 is given a flat prior, but we link the centroid to the weighted source position of the brightest pixels in $\mathrm{H}$ and S1A. The likelihood determined from the offset between these pixels and the predicted image positions of the S1 centroid (assuming one HSC-pixel positional uncertainty of $\sim 0.17$ ) are factored into the total likelihood. We then simultaneously vary the parameters of the lens and S1 using the light distribution of S2 as constraints. Using a pixelated source alone is unable to remove point-like residuals at the positions of the bright clumps in S2, so we also fit point sources convolved with the PSF at each of these seven locations (A-G in Figure 1), allowing their position and amplitude to vary. We also include a satellite galaxy (L1) parameterized as a singular isothermal sphere (SIS) at the main lens redshift between features A and C, as a smooth model is unable to account for these features. L1 is given a flat prior on its position and Einstein radius.

\subsection{Parameterized Source Modeling}

GLAFIC uses parameterized models for the extended light profile of the lensed source, contrary to GLEE. While the current public version of GLAFIC supports a single lens plane only, we recently implemented the ability to calculate the deflection angles over two lens planes using the standard multiplane lens equation formalism, which is essential for modeling of this system.

In principle, GLAFIC can fit the light and mass profile of the lens galaxies simultaneously. However, for simplicity, we model the light profile of the main lens galaxy (L) first with a Sérsic profile and subtract it from the $i$-band image. While holding the lens position fixed from the Sérsic model, we choose an elliptical power-law to model the lens potential.

We adopt the following strategy for modeling each lensed source. A single Sérsic model is used to describe the light profile of S1. The lens model includes an elliptical power-law 
for galaxy L and an SIS for the dark satellite L1, assumed to be at the same redshift as L. For S2, we need two-component Sérsic models to fit the compact (knots A, B, C, D, and E) and extended (blue ring) features. We also need another Sérsic component to model the knots $\mathrm{G}+\mathrm{F}$, which correspond to a distinct source (see Figure 2). For S2, the mass model is same as before $(\mathrm{L}+\mathrm{L} 1)$, except now there is a secondary lens plane to account for the mass of S1, which is modeled as an SIE. The center of the light profile of source $\mathrm{S} 1$ is the same as the associated mass component.

Both sources are modeled simultaneously but the likelihood of the lens model for $\mathrm{S} 1$ is calculated by masking out everything else in the image except $\mathrm{H}$ and S1A. Similarly, the likelihood of the lens model for S2 is calculated by masking out everything except the blue features. The final likelihood is a product of the two likelihoods. We use GLAFIC for solving the lens equations while the sampling of the posterior distribution of the model parameters is done via the Python module emcee (Foreman-Mackey et al. 2013).

\section{RESULTS AND SUMMARY}

The reconstructed image configurations are shown in the middle and right panels of Figure 3. We find that both GLEE and GLAFIC successfully reproduce the main features of the system. Both methods prefer a shallow mass profile $(\gamma \sim 1.5$, where $\rho \propto r^{-\gamma}$ ) for L, suggesting that there is a large dark matter fraction, possibly due to the cluster environment. Both models also require an additional mass component between images $\mathrm{A}$ and $\mathrm{C}$ to split this feature into two images. We have assumed that this mass component, which is undetected in the HSC imaging and FIRE spectroscopy, is at the same redshift as the lens galaxy, although it could potentially be a line-of-sight structure as well. The image splitting is unlikely to be due to S1 as the positions are not coincident. The velocity dispersion of $\mathrm{L} 1$, assuming an SIS profile, is $\sim 60-100 \mathrm{~km} \mathrm{~s}^{-1}$, although the uncertainties are large and there are likely degeneracies with the centroid position relative to features A and C. Deeper and higher-resolution imaging is needed to potentially detect L1.

Both models find that $\mathrm{S} 1$ has a velocity dispersion of $\sim 230 \mathrm{~km}$ $\mathrm{s}^{-1}$ (assuming an SIE profile) with moderate to high ellipticity. The GLEE model maps feature H to S1A-W, while S1A-S maps to a segment of $\mathrm{H}$ just above the lens galaxy, resulting in a source that either consists of two components or is highly elongated (e.g., an edge-on disk). The slight velocity offset of $\mathrm{H}$ and S1AW with S1A-S indicated by the FIRE data supports this interpretation. Unfortunately, the upper segment of $\mathrm{H}$ was not targeted in our FIRE observations. There are likely degeneracies among the physical properties of $\mathrm{L}$ and $\mathrm{S} 1$. More detailed mass models using improved data will explore these degeneracies and more accurately determine the model parameters.

By extrapolating the results from CFHTLS (i.e., one confirmed DSP lens from $\mathrm{Tu}$ et al. 2009), we expect to discover 10 DSP lenses in the HSC survey, of which Eye of Horus is the first. Our initial results highlight the potential wealth of information provided by DSP lenses, as well as the challenges of DSP lens modeling and the importance of highquality data and multiple lens model analyses to understand all of the degeneracies and systematic effects. The spectroscopic confirmation of both sources and the presence of the potential substructure causing the image splitting of features A and C make Eye of Horus a valuable system for studies of cosmology and galaxy structure.
We thank Aleksi Halkola for his contributions to this project. This work is based on data collected at Subaru Telescope, which is operated by the National Astronomical Observatory of Japan, and is supported by JSPS KAKENHI grant numbers JP15K17617, JP26800093, and JP15H05892. S.H.S. gratefully acknowledges support from the Max Planck Society through the Max Planck Research Group. We thank the referee for a helpful report. The Hyper Suprime-Cam (HSC) collaboration includes the astronomical communities of Japan and Taiwan, and Princeton University. The HSC instrumentation and software were developed by the National Astronomical Observatory of Japan (NAOJ), the Kavli Institute for the Physics and Mathematics of the universe (Kavli IPMU), the University of Tokyo, the High Energy Accelerator Research Organization (KEK), the Academia Sinica Institute for Astronomy and Astrophysics in Taiwan (ASIAA), and Princeton University. Funding was contributed by the FIRST program from Japanese Cabinet Office, the Ministry of Education, Culture, Sports, Science and Technology (MEXT), the Japan Society for the Promotion of Science (JSPS), Japan Science and Technology Agency (JST), the Toray Science Foundation, NAOJ, Kavli IPMU, KEK, ASIAA, and Princeton University. This paper makes use of software developed for the Large Synoptic Survey Telescope. We thank the LSST Project for making their code freely available. ${ }^{13}$ The Pan-STARRS1 (PS1) Surveys have been made possible through contributions of the Institute for Astronomy, the University of Hawaii, the Pan-STARRS Project Office, the Max-Planck Society and its participating institutes, the Max Planck Institute for Astronomy and the Max Planck Institute for Extraterrestrial Physics, The Johns Hopkins University, Durham University, the University of Edinburgh, Queen's University Belfast, the HarvardSmithsonian Center for Astrophysics, the Las Cumbres Observatory Global Telescope Network Incorporated, the National Central University of Taiwan, the Space Telescope Science Institute, the National Aeronautics and Space Administration under grant No. NNX08AR22G issued through the Planetary Science Division of the NASA Science Mission Directorate, the National Science Foundation under grant No. AST-1238877, the University of Maryland, and Eotvos Lorand University (ELTE).

\section{REFERENCES}

Alam, S., Albareti, F. D., Allende Prieto, C., et al. 2015, ApJS, 219, 12

Axelrod, T., Kantor, J., Lupton, R. H., \& Pierfederici, F. 2010, Proc. SPIE, 7740,774015

Barkana, R. 1998, ApJ, 502, 531

Blandford, R., \& Narayan, R. 1986, ApJ, 310, 568

Brammer, G. B., Sánchez-Janssen, R., Labbé, I., et al. 2012, ApJL, 758, L17

Bruzual, G., \& Charlot, S. 2003, MNRAS, 344, 1000

Calzetti, D., Armus, L., Bohlin, R. C., et al. 2000, ApJ, 533, 682

Chabrier, G. 2003, PASP, 115, 763

Collett, T. E., \& Auger, M. W. 2014, MNRAS, 443, 969

Collett, T. E., Auger, M. W., Belokurov, V., Marshall, P. J., \& Hall, A. C. 2012, MNRAS, 424, 2864

Cooray, A., Fu, H., Calanog, J., et al. 2011, ApJL, submitted (arXiv:1110.3784)

de Jong, J. T. A., Verdoes Kleijn, G. A., Kuijken, K. H., \& Valentijn, E. A. 2013, ExA, 35, 25

Dressler, A., Bigelow, B., Hare, T., et al. 2011, PASP, 123, 288

Edge, A., Sutherland, W., Kuijken, K., et al. 2013, Msngr, 154, 32

Falco, E. E., Gorenstein, M. V., \& Shapiro, I. I. 1985, ApJL, 289, L1

\footnotetext{
13 http://dm.1sstcorp.org
} 
Foreman-Mackey, D., Hogg, D. W., Lang, D., \& Goodman, J. 2013, PASP, 125,306

Gavazzi, R., Treu, T., Koopmans, L. V. E., et al. 2008, ApJ, 677, 1046

Ivezic, Z., Axelrod, T., Brandt, W. N., et al. 2008, SerAJ, 176, 1

Linder, E. V. 2016, arXiv:1605.04910

Magnier, E. A., Schlafly, E., Finkbeiner, D., et al. 2013, ApJS, 205, 20

Muzzin, A., Marchesini, D., Stefanon, M., et al. 2013, ApJ, 777, 18

Oguri, M. 2010, PASJ, 62, 1017

Oguri, M. 2014, MNRAS, 444, 147

Schlafly, E. F., Finkbeiner, D. P., Jurić, M., et al. 2012, ApJ, 756, 158

Schneider, P. 2014, A\&A, 568, L2
Simcoe, R. A., Burgasser, A. J., Schechter, P. L., et al. 2013, PASP, 125,270

Sonnenfeld, A., Treu, T., Gavazzi, R., et al. 2012, ApJ, 752, 163

Suyu, S. H., \& Halkola, A. 2010, A\&A, 524, A94

Suyu, S. H., Hensel, S. W., McKean, J. P., et al. 2012, ApJ, 750, 10

Suyu, S. H., Marshall, P. J., Hobson, M. P., \& Blandford, R. D. 2006, MNRAS, 371, 983

Tanaka, M. 2015, ApJ, 801, 20

Tonry, J. L., Stubbs, C. W., Lykke, K. R., et al. 2012, ApJ, 750, 99

Treu, T., Auger, M. W., Koopmans, L. V. E., et al. 2010, ApJ, 709, 1195

Tu, H., Gavazzi, R., Limousin, M., et al. 2009, A\&A, 501, 475 